\title{
LA DEMOCRACIA ATENIENSE
}

\section{ANTONIO GÓMEZ ROBLEDO}

INSTITUTO DE INVESTIGACIONES FILOSÓFICAS

Universidad Nacional Autónoma de México

Con excepción de la astronomía, obra de los caldeos, todo lo demás en el saber científico y las instituciones humanas, es a los griegos a quien lo debemos. Quedan por mil horizontes ini cómo negarlo? monumentos incomparables de otras culturas, pero son fruto de la empirie y no de la aplicación de un saber necesario y universalmente válido, en lo cual consiste la ciencia, según lo aprendimos del otro regiomontano ilustre. A todo esto presidió la razón, desde el día en que, según dijo Ortega y Gasset, los griegos se volvieron locos con la razón.

A partir de los presocráticos, el universo entero, la physis, como decían ellos, tuvo que justificarse ante la razón, y lo mismo ocurrió, a partir de Sócrates, con el hombre y la conducta humana. Hasta entonces, en efecto, a nadie se le había ocurrido que pudiera cuestionarse el mando y la obediencia, algo tan ineluctable y ciego como la ley natural. El faraón en su trono, y por debajo el escriba sentado escribiendo sus órdenes, y más abajo aún los fellah pendientes de sus decretos, como aquí, por debajo del tlatoani, la triste turbamulta de los macehuales. ¿A quién, una vez más, se le había ocurrido que el pueblo debe ser dueño de sí mismo, de su destino y del poder? A nadie en absoluto, hasta que por sí mismo también encontró este camino el pueblo elegido en su sentido más propio, el pueblo predilecto de la razón, del Logos, nombre que, andando el tiempo, dará san Juan a Jesucristo: "En el principio era el Logos".

No fue obra de un día, por supuesto, sino de muchos días y de muchos trabajos, según reza el título del poema hesiódico, Los trabajosy los días, donde por primera vez quizás (siglo VIII antes de nuestra era) se proclama la existencia del derecho, de un derecho común a todos, algo imbíbito en toda democracia genuina. "Las aves y los peces y las bestias, dice el poeta, se devoran entre sí, y es porque entre ellos no está la justicia. Mas el hijo de Cronos ha dado a los hombres la justicia (o el derecho, dike) y es con mucho lo mejor que tienen."

Son palabras, en el carmen hesiódico, no de un noble sino de un campesino, del pueblo llano, en suma, el cual desde aquella antigüedad tan remota, 
reclama un orden jurídico protector de sus derechos, como actualmente en la democracia moderna.

Contra la aristocracia emplaza su alegato Hesíodo, contra la aristocracia heredera de los reyes legendarios del Ática, en un proceso histórico que fue seguido luego en Roma: primero los reyes, en seguida los patricios y luego la república que resultó de la composición histórica entre patricios y plebeyos. En Grecia, más o menos, otro tanto, primero los reyes, luego los eupátridas, y por fin, pura y simplemente, el demos. Lo que no quiere decir que se abolieran las viejas magistraturas, entre ellas y a la cabeza el areópago, el venerable cuerpo que tenía su sede en la colina de Ares (de ahí su nombre) y ante el cual pronunciará un día san Pablo su célebre homilía en defensa del dios desconocido. Todo aquello subsiste, y no sólo el areópago sino hasta el arconte rey, que de tal no tiene sino el nombre, y que no es otra cosa que el juez de instrucción ante el cual comparece Sócrates al ser emplazado a responder del crimen de impiedad.

Nova et vetera. Lo mismo pero distinto, porque todo el poder va a estar en adelante no en manos de la nobleza sino en manos del pueblo, del demos, al que vemos ya encabritarse desde los poemas homéricos, aunque con la consiguiente indignación del poeta, eco del desprecio que los héroes divinos, Agamenón y Aquiles, sienten por la masa popular.

Esta última, empero, es la que a la postre triunfa, a través de las reformas sucesivas de Solón, Arístides, Clístenes, Efialtes y Pericles. Atenienses todos ellos, porque la democracia, lo que hoy entendemos por este nombre, no es una creación helénica, así en general, sino específicamente una creación ateniense. Todo puede ponerse a discusión, menos el hecho histórico e indiscutible de que la democracia nació en Atenas.

Dicho lo anterior, y antes de seguir adelante, habrá que hacer ciertas precisiones, porque no se crea que la democracia ateniense, con ser su paradigma, guarda un paralelo exacto con las democracias modernas, ya que mi propósito no ha sido el hacer un panegírico desaforado de la democracia clásica.

Todo el pueblo, acabamos de decir, toma parte en el gobierno, sólo que por pueblo debemos entender, en la ciudad antigua, no todos los nacidos en determinado territorio, sino únicamente los nacidos libres y no esclavos, y nacidos, además, en Atenas por lo menos, de progenitores atenienses, los dos, lo cual significa la aplicación conjunta del ius sanguinis y del ius soli, y no como actualmente, en la constitución política mexicana, donde la nacionalidad se recibe por una u otra vía, iure sanguinis o iure soli, y en el primer caso de un solo progenitor en último extremo.

A los esclavos nativos habrá que sumar aún los extranjeros avecindados, los metecos, por lo que de la población del Ática, evaluada en medio millón de habitantes en números redondos, no quedan, deducidos esclavos y metecos, siendo 120 mil ciudadanos, de los cuales aún habrá que restar las mujeres y 
los niños para quedarnos al fin con treinta mil ciudadanos en quienes recae, en última instancia, el ciudado de la república.

De conformidad con estos cálculos, la democracia viene a quedar al fin en pocas manos, y más aún si se toma en cuenta que los grandes fundadores de la democracia fueron casi todos miembros de la antigua nobleza, notoriamente los dos últimos que imprimieron su último sello a la reforma constitucional, Efialtes y Pericles, pertenecientes ambos a la estirpe de los Alcmeónidas. Y con respecto al último de los nombrados, a Pericles, quien dio su nombre a su siglo, como lo hicieron, cada uno con el suyo, Augusto y Luis XIV, fue por más de quince años el supremo titular del poder (strategos autokrátor) un poder absoluto y exclusivo, aunque refrendado libremente cada año por el voto popular. Cuando, en cambio, gobierna genuinamente la masa, lidereada por un curtidor, Anito, uno de sus primeros actos es la condenación de Sócrates, cuyos acusadores han sido el propio Anito, con otros dos achichincles tan mediocres como él mismo. De esta mancha no ha podido liberarse jamás la democracia ateniense, y por más que en la literatura más reciente no falten los estudios enderezados a justificar aquel histórico veredicto. Dícese que tuvo que ser el que fue, porque Sócrates censuraba acremente la democracia, a lo cual lo único que por nuestra parte nos permitimos observar es que la primera virtud de la democracia ha de ser la tolerancia, la que ha de tener con las opiniones adversas a su credo, en lugar de hacer beber la cicuta, o su equivalente, a sus opositores.

Con todo ello, sin embargo, continúa siendo hasta hoy un gran acontecimiento histórico la proclamación de un régimen abierto a todos los hombres libres en general, la carrera abierta a todos los talentos, a dicho de Napoleón I (la carrière ouverte à tous les talents), o como se dirá aún, mucho más tarde, el gobierno del pueblo, por el pueblo y para el pueblo.

Esto último, el que la democracia deba ejercerse precisamente en beneficio del pueblo (ya que sería absurdo que el pueblo actuara, conscientemente por lo menos, en su maleficio) muestra bien a la clara que la democracia está muy lejos de ser simplemente una teoría de la titularidad del poder público, una formalidad vacía en fin de cuentas, sino que tiene un contenido material bien definido y concreto, como lo es el bienestar de la sociedad en todos los órdenes, el mayor bien para el mayor número, the greatest good for the greatest number, según reza el apotegma atribuido a Bentham. Ahora bien, todo esto estaba dicho ya desde entonces, y por la mayor autoridad política en aquel momento, es decir por el propio Pericles.

El término demacracia ( $\delta \eta \mu \kappa \rho \alpha \tau i \alpha)$ es del siglo V, el siglo de Pericles, y aunque su invención, según se cree hoy, parece ser más bien de los sofistas antes que de los filósofos y los políticos, el hecho es que el primer documento donde lo encontramos es en la historia de la guerra del Peloponeso 
de Tucídides, en el célebre Epitafio de Pericles, en su oración fúnebre, pronunciada en el cementerio del Cerámico, por los caídos en el primer año de la guerra del Peloponeso. En este discurso, pues, y bien consciente de que están apenas en los pródromos de una guerra larga y difícil, trata Pericles de templar el ánimo de su auditorio con la consideración de que los muertos (y los morituros que vendrán tras de ellos) han caído por la preservación de la ciudad cuyo prestigio le viene, entre otras cosas, del régimen político que profesa, y que es la democracia. "Llámase a este gobierno democracia, continúa diciendo Pericles, porque no mira al bien de pocos, sino al del mayor número" (Tuc., II, 37). Por conservar este régimen y legarlo a los hijos, bien vale el sacrificio de la propia vida.

A renglón seguido, y con esto terminaré mi presentación de la democracia clásica, la oración fúnebre alude al tercer carácter constitutivo de la democracia, que es la igualdad de todos ante la ley: la isonomía, y es una lástima que el término no haya trasmigrado a nuestro idioma, como sí lo ha hecho, en cambio, al francés. La democracia, dirá más tarde Aristóteles, es el pacto social entre hombres libres e iguales, ante la ley se entiende.

Cada ateniense es libre, dice aún Pericles, para vivir a sus anchas, a su gusto ( $\left.\kappa \alpha \theta^{\prime} \dot{\eta} \delta o \nu \dot{\eta} \nu\right)$, mas con obediencia, añade en seguida, "a los magistrados y a las leyes cuyos órganos son aquéllos, sobre todo a las leyes que protegen a los oprimidos".

En estas palabras está cifrado lo que hoy llamamos Estado de derecho, es decir la subordinación a la ley por parte de gobernantes y gobernados, y con mayor celo aún, si cabe, por parte de los primeros, ya que si el particular puede hacer lo que quiera, menos lo que la ley le prohíbe, el gobernante, en cambio, no puede hacer sino aquello a que la ley lo autoriza, según la doctrina uniforme, a lo que creo, en derecho constitucional y en derecho administrativo.

De esta alabanza de la ley (praise of law) según reza el título del hermoso ensayo de Werner Jaeger, está llena la literatura helénica comenzando por el celebérrimo fragmento de Píndaro, "la ley es el rey de todas las cosas" ( $\nu$ ó $\pi \dot{\alpha} \nu \pi \nu \nu \beta \alpha \sigma \iota \lambda \epsilon \dot{v} \varsigma)$, y en seguida, o al mismo nivel tal vez, la decisión de Antígona de dejarse enterrar viva con tal de no transgredir las leyes no escritas e inviolables de los dioses, y por último, la decisión de Sócrates de no apelar a la fuga que le ofrecen sus amigos, por no violar las leyes de la ciudad en virtud de las cuales ha sido condenado a muerte.

Por encima del pueblo soberano estaba la soberanía de la ley, por encima de todos en absoluto; y esta tradición, aunque frecuentemente transgredida en los hechos, no fue jamás contradicha en el derecho sino a partir del siglo XVI, cuando Jean Bodin, en su obra De republica, define la soberanía como el poder supremo superior a las leyes, por estar el gobernante exento de ellas: summa in cives ac subditos legibusque soluta potestas. Es, en otro giro, el princeps legibus solutus, contra lo cual reaccionará con gran vigor la escuela española del mismo 
siglo XVI, Francisco de Vitoria a la cabeza, quien prodamará altamente que las leyes obligan también a los reyes: leges obligant ipsum regem.

Volviendo a Grecia, y para acabar de configurar en ella el imperio de la ley, no podemos pasar por alto, así no sea sino por haber sido el más lejano antecedente de nuestro juicio de amparo, la institución ateniense del recurso de

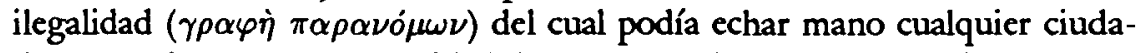
dano para impugnar la ilegalidad de una ley cualquiera que estuviera en contradicción con la ley constitucional $(\pi o \lambda \iota \tau \epsilon i \alpha)$ o con una ley ordinaria que no hubiera sido derogada, o cuando, por último, la ley nueva adolecía de un vicio formal, como lo era el no haber sido propuesta a la asamblea $(\xi \kappa \kappa \lambda \eta \sigma i \alpha)$ por el senado $(\beta o v \lambda \eta ́)$ del cual debía partir inexorablemente la iniciativa de las leyes. De esta suerte, la acción popular de ilegalidad, la $\gamma \rho \alpha \varphi \dot{\eta} \pi \alpha \rho \alpha \nu o ́ \mu \omega \nu$, escribe Gustave Glotz, fue la defensa principal de la constitución democrática, la cual, como se ve, estaba temperada por el principio aristocrático en la institución del senado, y de hecho, aunque no de derecho, por el principio monárquico, como en el largo mando de Pericles. Por algo dirá Aristóteles que la mejor constitución es la que resulta de la mixtura de los tres principios, y lo declara más de propósito en el equilibrio de los tres poderes, cuya paternidad, simplemente por no leer los textos clásicos, se atribuye erróneamente a Montesquieu.

En estos caracteres, en suma, en el ejercicio directo del poder político, en la administración pública en beneficio de los ciudadanos, en la libertad de pensamiento y de palabra, y en la sujeción a la ley por gobernantes y gobernados, veo yo la imagen que me he formado de la democracia antigua.

Lo último que se me ofrece decir es que ya desde el nacimiento de la democracia, o de su consolidación si se quiere, no faltaron en Atenas críticos muy concienzudos de la nueva forma de gobierno, y no sólo entre la aristocracia, donde era de esperarse, sino aun en la clase media, a la cual, a primera vista por lo menos, venía de perlas la democracia. Era el caso de Sócrates, como es bien sabido, el cual, y no por interés político sino porque así lo sentía, no fue nada parco en su crítica de la democracia, aunque no por esto, huelga decirlo, mereció la muerte.

En sentir de Sócrates (por aquí más o menos iban sus razonamientos), la política es un arte, el de mayor responsabilidad y el más arduo, y ha de estar, por consiguiente, en manos de expertos y no del hombre de la calle, como era el caso desde la reforma de Efialtes, al que por algo mandó asesinar la nobleza.

A Sócrates, según lo dijo mil veces, le parecía inconcebible que así como no confiamos nuestra casa, nuestra salud y nuestra navegación sino al arquitecto, al médico y al piloto con mayor conocimiento y experiencia, así por el contrario dejamos la dirección del Estado al arbitrio de la masa iletrada que procede a llenar las vacantes de las magistraturas unas veces por sorteo, y otras, en el

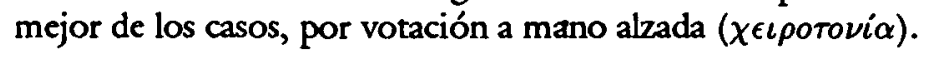


Ahora bien, y si teóricamente podemos dar la razón a Sócrates (podemos y no debemos, lo subrayo) en la práctica, sin embargo, en la larga práctica histórica desde entonces hasta hoy lo que hay es que el poder personal, irresponsable y perpetuo, ha sido siempre víctima de la corrupción y ha degenerado siempre en tiranía, con la catástrofe final, a corto o largo plazo, del régimen y del pueblo. Ha sido el caso, en nuestros días, del fascismo y el nazifascismo, y lo fue, en la Grecia clásica, con la reacción antidemocrática del tirano Pisístrato y sus sucesores. Pisístrato, hasta donde sabemos, embelleció a Atenas con grandes obras y se comportó con moderación y respeto para con sus conciudadanos. Sus hijos, sin embargo, Hipias e Hiparco, los pisistrátidas, se comportaron de modo muy distinto, y quienes se alzaron para asesinarlos, Harmodio y Aristógiton, fueron acreedores al supremo dictado de libertadores. Por todo lo cual nos limitamos a refrendar el veredicto del historiador Rex Warner en las siguientes palabras:

La libertad de un hombre es más valiosa para él que cualquier orra cosa. Ninguna eficiencia del gobierno puede compensar su pérdida. En la democracia, como en todas las cosas, hay algunas fallas. Sus procedimientos pueden ser lentos y engorrosos; pueden tomarse decisiones equivocadas bajo la influencia de alguna súbita tormenta emocional, en la que no se presta oído a los mejores. Estas son deficiencias inherentes a la naturaleza de la democracia; pero son mucho menos serias que los peligros que entraña el conferir poderes sin restricción a una minoría. Ningún sistema de gobierno es perfecto y, dado que el hombre es lo que es, ninguno puede serlo. Pero la democracia posee la inestimable ventaja de garantizar la libertad, al paso que todas las demás formas de gobierno, por eficientes que sean, tienden hacia la dirección contraria. Por lo tanto, la democracia, con todos sus defectos, es el menos malo de todos los sistemas de gobierno hasta ahora concebidos por el hombre. (R. Warner, Pericles el ateniense, Barcelona, 1989, p. 94.)

\section{La democracia en su proyección internacional}

Hay otro debate aún más amplio, por lo menos en su dilatación históricogeográfica, y es el que se abre, de Atenas a nuestros días, con la proyección de la democracia al mundo de la política exterior. El problema, más en concreto, es el de si la democracia tendrá que ser fiel a sí misma en su vida de relación, guardando hacia todos sus horizontes un orden de paz y de justicia, o si, por el contrario, serán los valores opuestos, disvalores mejor dicho, los que encarne en su conducta internacional, con los pueblos débiles por lo menos. Ha sido el caso, para no ir más lejos, de los Estados Unidos y del Reino Unido, democracias ejemplares en su régimen interno, y adalides máximos del imperialismo y subyugación de los pueblos en su política exterior.

En Atenas fue lo mismo y desde la primavera de la democracia. Queriendo hacer de Atenas una gran potencia -el más justo de los desiderata y la primera 
norma de seguridad, tanto frente a Persia como frente a Esparta- Pericles fortaleció la liga que ya existía, y que había sido creada por Arístides, entre Atenas y otras ciudades marítimas, una confederación, en otras palabras, cuya sede central estaba en Delos, isla central de las cícladas, y que merecía este honor no sólo por su posición central sino por haber sido la cuna de Apolo, el dios de la luz y de la inteligencia. Atenas, sobra decirlo, tenía un papel de primera importancia en esta confederación talásica que se alzaba frente a la geocracia espartana, pero jurídicamente no era sino un primus inter pares en el consejo que cada año se reunía en Delos para decidir, por voto libre y mayoritario, sobre la política general de la confederación.

Ahora bien, y si así fue con Arístides y sus sucesores inmediatos, todo mudó en el "gran cambio", como dice Bowra, el que efectúa Pericles en su función, renovada por tantos años, de strategos autokrátor. Bajo su gobierno, en efecto, la liga se transforma gradualmente en una agencia de Atenas, en su servicio y beneficio en buenas palabras. Lo primero, y es algo de enorme trascendencia, es el traslado del tesoro federal, de Delos a Atenas, con el pretexto, por lo demás muy digno de atención, de que la isleta de Delos podía ser fácilmente presa de fuerzas médicas o espartanas, o simplemente de piratas. Hasta aquí, una vez más, era atendible la mudanza del tesoro, pero lo grave estuvo en que luego se dispuso de él, o sea de las cuotas de los miembros de la liga, no en beneficio común, como era lo debido, sino en el interés exclusivo de Atenas. Pericles, huelga decirlo, no distrajo para su bolsillo ni una dracma del tesoro, pero sí lo aplicó a la construcción de las obras, las de la Acrópolis sobre todo, que son hasta hoy la mayor maravilla del mundo. Fue algo semejante a lo que haría más tarde el papa León $\mathrm{X}$, al aplicar el dinero de las indulgencias no al incremento de su propio patrimonio (no le era necesario a un miembro de la familia de los Médicis) sino a la construcción de la basílica de San Pedro, sin lo que no tendríamos hoy la estupenda conjunción de Miguel Ángel y el Bernini en la plaza más hermosa del mundo. Váyase, pues, lo uno por lo otro, y hoy pademos regocijarnos tanto del tráfico de indulgencias como del traslado del tesoro federal a la Acrópolis.

Lo que, en cambio, no puede ya cohonestarse así como así, por no estar ya nimbado de belleza, fue la política de abierta expansión e imperialismo que siguió Pericles en su transformación de la liga interaliada. Expliquémonos.

La expansión, en primer lugar, consistió sobre todo en la creación de las llamadas cleruquías, que eran lotes o parcelas de tierra que fueron asignadas en otras islas a ciudadanos atenienses, por la mayor parte menesterosos o desempleados. Para ellos estaba muy bien, por supuesto, y para Atenas también, que reducía en algo de este modo su plétora ecológica, de indigentes sobre todo, mas no para las ciudades donde se instalaban las cleruquías, cuyos propietarios, los atenienses transterrados, hacían sentir su presencia opresora en su nuevo domicilio. A poco andar, en efecto, y con el apoyo de la metrópoli, 
se convertían de colonos en amos. Fue el precedente más genuino, a lo que pienso, de las colonias que andando los años, o los siglos, tuvieron los países europeos en otros continentes, y que fueron, como es manifiesto, la impronta de su dominio en el exterior.

La implantación de este régimen dentro de la liga marítima señoreada por Atenas, provocó en numerosas islas, como era natural, un movimiento de desafección y a la postre de segregación de la liga. Ahora bien, a esto último no pudo nunca avenirse Atenas, por lo que resistió siempre a cualquier movimiento secesionista, como si en la Acrópolis imperara otro Lincoln. Ni sólo esto, sino que en ocasiones se propasó Atenas hasta obligar por la fuerza a entrar en la confederación a los Estados que lo repugnaban y que no podían quedar fuera por su posición estratégica. Fue el caso, entre otros, de la isla de Melos (416 A. C.) donde el ejército ateniense, al desembarcar victorioso, pasó a cuchillo a toda la población masculina en edad militar, y al resto, mujeres y niños, los redujo a la condición de esclavos. Sin tanto salvajismo, algo semejante pasó con nosotros en Veracruz (1914) donde el presidente Wilson pretendió enseñarnos la democracia a cañonazos.

Probablemente Pericles, fallecido hacía años, no habría ordenado o tolerado desmanes como los de Melos et alia similia, pero sí había sembrado la simiente que dio luego tan abominables retoños. Pudo darse por tanto, ya desde entonces y hasta nuestros días, una democracia beligerante y agresiva, por grandes que sean sus virtudes cuando sólo se atiende al régimen interior. Pericles mismo, aún en pleno poderío, había desplegado una política de la mayor dureza en la guerra de Samos, uno de los miembros más vigorosos e importantes de la confederación. La ocasión de la guerra fue la disputa entre Samos y Mileto, otro miembro de la liga, por la posesión de Priene. Pericles decidió intervenir en favor de Mileto, y no tanto porque tuviera de su parte la razón, sino sobre todo, como lo asegura formalmente Plutarco, por complacer a Aspasia, la bella cortesana milesia que se había enseñoreado totalmente de su alma y de su cuerpo. Ya en campaña, después de un bloqueo de nueve meses, sostenido por una flota ateniense de 200 trirremes, Samos hubo de capitular al fin. Entre las cargas que los vencidos hubieron de llevar sobre sí, estuvo la demolición de sus murallas, la destrucción de su armada, el pago de 1500 talentos y la obligación de contribuir periódicamente con un contingente de soldados al ejército ateniense.

No en vano había llevado Pericles a su clímax la democracia, porque cuando regresó a Atenas rodeado de los aplausos de la multitud y lloviéndole sobre la frente flores y laureles, no faltó quien le murmurara al oído, parece haber sido una mujer, palabras como éstas: "Sí, Pericles, todo esto es maravilloso y digno por cierto de muchas coronas, quiero decir el haber inmolado a tantos y tan bravos ciudadanos, pero no en guerra contra los fenicios ni contra los medos, sino para abatir una ciudad aliada y hermana". 
Todavía alcanzó Pericles a saborear su gran momento, el de su apogeo, cuando al frente de una armada imponente anduvo por el Mar Negro, entre Tracia y el Quersoneso, "democratizando" aquellas regiones y sembrando por doquiera más y más cleruquías. No alcanzó a ver, sin embargo, que el fin estaba cerca, para él y para Atenas. No porque faltara nada, ni en el tesoro ni en el ejército ni en la flota. Era sencillamente porque saltaba a los ojos de todos la contradicción en que reposaba el Estado ateniense hacía buen número de años, entre el liberalismo de sus principios en el interior y su conducta despótica en el exterior. Todos sus aliados vivían entre el temor y el terror; ahora bien, a todo terror acaba por llegarle su termidor.

El de aquel momento histórico fue la guerra del Peloponeso, cuya responsabilidad (aitia) la atribuye Plutarco, sin el menor miramiento, a Pericles, y fundamenta su dicho en los dos capítulos siguientes.

El primero, el odio personal que Pericles sentía por los megarenses, y que le llevó a hacer aprobar un decreto por virtud del cual eran excluidos aquellos de todos los puertos y mercados de Atenas. Ahora bien, comenta Bury, siendo Mégara un país enclavado, el decreto significaba su ruina completa, y no hay que olvidar, además, que Mégara era un miembro importante de la liga del Peloponeso señoreada por Esparta. En una guerra entre la liga espartana y la confederación ateniense se traduciría pues, tarde o temprano, el decreto ateniense contra Mégara. Ahora bien, Pericles era, por supuesto, el primero en saberlo, pero creía, o aparentaba creer, que si cedía al primer requerimiento, luego tendría que hacer lo mismo con todos los que luego seguirían indefectiblemente, por lo que se mantuvo inflexible en la persistencia del decreto antimegárico, con lo que, conscientemente, precipitó la guerra.

El segundo motivo, y el más poderoso tal vez, tal como lo presenta Plutarco, en la decisión belicista de Pericles, fueron los grandes embrollos en que al final de su vida se vio metido por causa de la persecución de que fueron objeto sus amigos más íntimos, y que no se habrían producido si no hubiera estado decreciendo, en una especie de razón directa, la popularidad de Pericles. Fue en primer lugar Fidias, el maravilloso artista del Partenón y demás monumentos del Acrópolis, quien acabó en la cárcel, de enfermedad, dice Plutarco, aunque luego agrega que, según otros, fue por veneno. Ahora bien, en opinión de Aristófanes, "el origen de nuestros infortunios fue la desgracia de Fídias. Pericles temió compartir su suerte, y para prevenir este peligro personal, lanzó el decreto contra Mégara, inflamó la Ciudad y alentó la guerra, esta guerra cuya humareda ha arrancado lágrimas de los ojos de todos los griegos" (La paz, 605 ss.).

Viene luego la acusación de impiedad (asebia) en contra de Aspasia, imputación que hasta hoy no sabemos qué fundamento pudo tener; serían tal vez las conversaciones libres que sobre religión y filosofía se tenían en casa de Pericles. Sea de ello lo que fuere, lo cierto es que Pericles hizo ante los jueces 
la tragicomedia que era entonces usual, "derramando copiosas lágrimas" para obtener la absolución de la acusada, lo que no habría hecho si hubiera estado todavía realmente en el poder.

Del proceso semejante intentado contra su otro huésped ilustre, el filósofo Anaxágoras, sale Pericles más o menos airoso, pues a tiempo pone en cobro al filósofo mandándolo a Lámpsaco, donde terminó sus días en paz y rodeado de honores.

En cuanto a Pericles, dándose bien cuenta, por todos estos incidentes, de que el golpe siguiente sería contra él, no se le ocurrió nada mejor que atizar la discordia internac:onal y encender el fuego de la guerra, en la creencia de que con una victoria rápida podría rehacer su prestigio personal y la seguridad inviolable de Atenas. "Tales fueron, termina diciendo Plutarco, los motivos por los cuales no permitió al pueblo hacer concesiones a los lacedemonios. La verdad, sin embargo, está aún oscura." (Vida de Pericles, 32) Hermosa confesión, por cierto, del historiador filósofo.

En la conducción de las hostilidades, por todo cuanto sabemos, Pericles fue igualmente de mal en peor. En la guerra a muerte que empezaba entre la talasocracia ateniense y la geocracia espartana, Pericles, no pudiendo obviamente combatir a su rival por tierra ni cercarla por mar (a sus satélites sí, por supuesto, como a Corinto) optó por el peor partido, que fue, ante el avance del ejército espartano, encerrarse en Atenas, y no sólo con la población residente, sino con toda la población del Ática prácticamente, hombres, mujeres y niños, que no quisieron ser presa de los soldados lacedemonios que irrumpían y talaban cuanto veían. Amontonados así en la capital, unos sobre otros y en miserables tugurios, no tardó en caer sobre ellos la peste, la terrible peste que duró cuatro años y entre cuyas víctimas estuvieron Pericles y dos de sus hijos.

Así deshizo Pericles en sus años postreros todo cuanto había hecho en los primeros, lo que prueba una vez más lo azaroso y frágil que es la política y el político que la encarna, el cual de repente pierde su genio y no hace en adelante sino dar traspiés. Algo semejante, dicho sea de paso, a lo que está pasando con Gorbachev, quien después de haber brillado como el astro cumbre de la nueva democracia, la democracia socialista, a lo mejor termina como el destructor del imperio soviético y de la organización política que lo encarna.

Para nosotros, en fin, que no tenemos por qué juzgarlo como político, somos deudores a Pericles por habernos enseñado la democracia como régimen de gobierno y como forma de vida, aquella en la cual fueron construidos los monumentos de la Acrópolis, de los cuales emana, y posiblemente sea su mayor encanto, un mensaje perenne de libertad. Son eternos porque reúnen en sí todas las dimensiones temporales, conforme al famoso pasaje de Plutarco:

Cada uno de ellos, no bien acabado, era tan bello que tenía ya la pátina de lo antiguo, $y$ tan perfecto que ha guardado hasta hoy la frescura de una obra reciente, 
a tal punto florece en ellos una eterna juventud, la que les ha preservado de la injuria del tiempo. Parecería como si estas obras tuvieran en ellas un soplo siempre vivo y un alma inaccesible a la vejez.

En cuanto a la democracia como forma de vida, el bomo democraticus, como si dijéramos, lo encontraremos siempre en el Epitafio de Pericles, el discurso que pronunció en el cementerio del Cerámico en homenaje a los caídos en el año primero de la guerra del Peloponeso. La idea central del discurso es presentar una imagen espiritual de Atenas de valor tan alto como para motivar la convicción de que por esta ciudad, por preservarla en su esencia, está bien el sacrificio de los que cayeron en su defensa. "Nuestra ciudad, dice Pericles, es la escuela de Grecia (y luego del mundo) y creo que cualquier ateniense puede lograr una personalidad completa en los más distintos aspectos y dotada de la mayor flexibilidad y buena gracia." (Tuc., II, 41)

Por todo el discurso circulan ideas como estas, y acaso su mejor concentrado esté en la tan conocida y doble sentencia que resume el perfecto equilibrio entre la inteligencia y el sentimiento:

Amamos la belleza sin dispendio y el saber sin molicie:

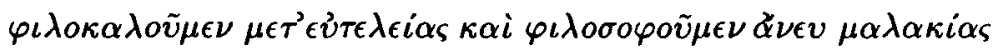

A uno de los capítulos de La democracia en América, su autor, Alexis de Tocqueville, le puso el título siguiente: "Por qué el estudio de la literatura griega y latina es particularmente útil en las sociedades democráticas". Como verá el que lo leyere, lo que quería Tocqueville es que las democracias modernas se nutran de la democracia antigua, de los textos sagrados de la libertad. Tal ha sido mi modesto propósito al presentar al lector un manojo de ellos, que son, según las palabras de Tucídides, "un tesoro para siempre". 